\title{
Performance Evaluation of WPAN MAC Protocol with Improved Backoff Algorithm
}

\author{
S. Mehta (Corresponding Author) \\ UWB Wireless Communications Research Center, Inha University, \\ Incheon, 402-751, Korea
}

K. S. Kwak

UWB Wireless Communications Research Center, Inha University, Incheon, 402-751, Korea

\begin{abstract}
The wireless personal area network (WPAN) is an emerging wireless technology for future short range indoor and outdoor communication applications. The IEEE 802.15.3 medium access control (MAC) is proposed, especially, for short range high data rates applications in WPAN, to coordinate the access to the wireless medium among the competing devices. A thought of an improved backoff algorithm (IBA) was brought up in [3]. In this paper, we propose an IBA for contention process of IEEE 802.15.3, where binary exponential backoff (BEB) is originally used. Here, we compare both BEB and IBA algorithms, and show the superiority of IBA over BEB in terms of channel efficiency, channel access delay and energy efficiency.
\end{abstract}

Keywords: WPAN MAC Protocol, Performance Analysis, Backoff Algorithm 


\section{Introduction}

The IEEE standard 802.15.3 MAC or WPAN MAC layer [1] is based on a centralized, connection oriented topology which divides a large network into several smaller ones termed “piconets”. A piconet consists of a Piconet Network Controller (PNC) and DEVs (DEVices). One DEV is required to perform the role of PNC (Piconet Coordinator), which provides the basic timing for the piconet as well as other piconet management functions, such as power management, Quality of Service (QoS) scheduling, security, and so on. The standard also allows for the formation of child piconets and neighbor piconets. In IEEE 802.15.3 MAC protocol, the channel time is divided into superframes, where each superframe beginning with a beacon. The superframe is composed of the three major parts: the beacon, the optional contention access period (CAP), and the channel time allocation period (CTAP) or channel time allocation time (CTA). Wireless channel is usually vulnerable to errors. Hence, error control mechanism is an essential part of any MAC protocol design. In accordance with that, IEEE 802.15.3 standard defines three types of acknowledgment mechanisms for CTAs and CAPs : the No-ACK, Imm-ACK, and Dly-ACK mechanisms [1].

During the CAP time devices request for reservation in CTA and also send data packets if needed. So the time length of CAP is dynamic and determined by the PNC. The longer the duration of CAP time is, more the number of devices will send their CTA slot requests and causes less time for CTA slots. Hence, it is important to improve the performance for both data and request packets transmission within limited time frame of CAP. In our previous work [2] we present the detail performance analysis of WPAN MAC and identified some key issues to improve the MAC performance, especially, for CAP duration. In this paper, we limit our research focus on improving the performance of CAP using IBA instead of BEB during the contention process, and hence, overall WPAN MAC performance. During the CAP, MAC protocol performs back off procedure before transmitting any kind of data or request packets. This backoff mechanism is similar to CSMA/CA mechanism of IEEE 802.11 with some different parameters. In WAPN MAC, retry count is limited up to 3 counts ( 0 to 3 ) with maximum window size of 64 slots [7, 15, 31, and 64]. It is worth to note that traditional ${ }^{1}$ backoff method has the following disadvantages.

- Traditional back-off takes time for CW (Contention Window) to correctly adapt to the right value when the active population (of nodes) is large.

- If $\mathrm{CW}$ is already large then the latency performance is high.

- A station that successfully transmits resets its CW to a small, fixed minimum value of CW. Consequently, the node has to rediscover the correct CW, wasting some precious bandwidth.

- Traditional backoff algorithm could not take the advantage of shared learning when number of nodes is increasing.

- Traditional back-off‘s success probability degrades with increasing number of nodes.

\footnotetext{
${ }^{1}$ Here, traditional backoff procedure means CSMA scheme with binary exponential backoff (BEB), unless and otherwise specified.
} 
- Traditional back-off is also not suitable for power saving networks application.

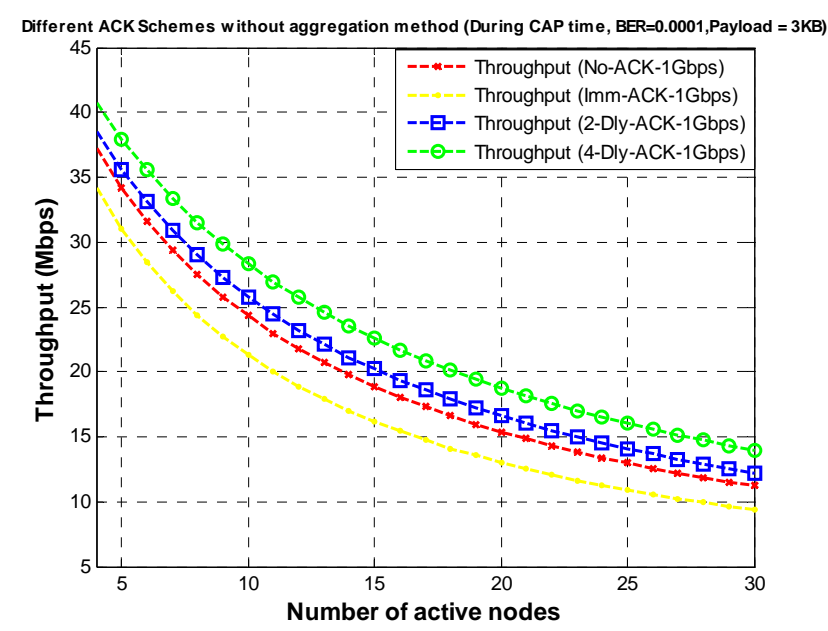

Figure 1 Throughput versus number of active nodes (This result is taken from [2])

Our results from [2] also support the aforementioned disadvantages. Figure 1 shows the normalized throughput for different number of active nodes. It can be seen that throughput decreases as the number of active nodes increases due to increase in collision probability and channel access time.

In nutshell, during the CAP, backoff window size and the number of active nodes are the major factors to have impact on the throughput performance. These observations and result lead us to use new medium access scheme for IEEE 802.15.3 networks. So we adopted the basic idea of geometrically increasing distribution from [3] and applied to IEEE 802.15.3 as IBA. Even though the basic idea of IBA is adopted from other research work the main contributions of this paper are as follows.

- To apply the geometrically increasing distribution as IBA to IEEE 802.15.3 MAC.

- $\quad$ To present a comparison of BEB and IBA for a medium access scheme.

- To present the detail performance evaluation study in terms of channel efficiency, channel access delay and energy efficiency to compare BEB and IBA.

To the best of our knowledge, there is no work on the performance or channel analysis of IEEE 802.15.3 networks with respect to contention based scheme during the CAP time. However, a large amount of literature is available on IEEE 802.15.3 MAC scheduling, optimization of superframe size, and various traffic analyses. Similarly, most of the studies on backoff algorithm (BA) are on the stability issue rather than performance analysis of BA. Some of the important related works on BA are as follows

In [4] authors, presented the performance analysis of exponential BA with different retry limit. The main contribution of their work is to present a new and efficient analytical method to evaluate the performance of a network with exponential BA. Furthermore, their work laid a good foundation work in analytical study of IEEE 802.11. In [5] authors compared the different approaches for binary exponential BA and study the impact of the 
stochastic property of the backoff time interval on the performance. In [6] authors, presented different backoff algorithms and compared them with standard exponential BA. Their simulation results show the better throughput, resolution efficiency and better stability over exponential BA. In [7] authors, compared five different BAs in multihop wireless scenarios and proved that none of the existing BAs are capable of handling multihop wireless scenarios

In this paper our main focus is to analyze and compare the performance of both IB and BEB with respect to network load in steady state condition. This paper, in our knowledge, is the first attempt to present the performance results of IBA in the contention process of IEEE 802.15.3 with those of BEB during the CAP time.

The rest of the paper is outlined as follows. In section 2 we describe the BEB and IBA medium access schemes. In section 3, we present the performance evaluation of BEB and IBA, and finally, conclusions and future work are drawn in section 4.

\section{BEB and IBA Schemes}

BEB Scheme: BEB based carrier sense multiple access with collision avoidance (CSMA/CA) is the most basic and widely used mechanism for MAC protocol. In WPAN MAC, if the channel is idle for backoff inter frame space (BIFS) or short inter frame space (SIFS) the $\mathrm{DEV}^{2}$ start executing the BEB scheme. At each packet transmission, the back off counter is uniformly selected from the given range of [0, CW-1]. Here, CW is known as contention window and its value depends on the number of failed transmissions for the packet. At the first transmission attempt, CW is set to minimum value of 8 and if transmission attempt fails then its value gets double, and again set to minimum value on successful transmission. In WPAN MAC, the maximum contention window value is set to 64 with the maximum retry limit up to 3 counts. After selecting CW node decrement its value by 1 as long as channel is sensed idle and freeze its value when channel is sensed busy.

By using BEB method MAC protocol attempt to adapt to the active population size of contending DEVs. However, this method has several disadvantages which we have already discussed in the last section. This approach does not work well when we are interested in the high bandwidth utilization, latency, and energy efficiency. To improve the channel performance during the CAP time, we use a fixed-size contention window, but a non-uniform, geometrically-increasing probability distribution for picking a transmission slot in the contention window interval.

IBA Scheme: In contrast to BEB scheme, IBA scheme uses a small and fixed CW. In IBA scheme, nodes choose non-uniform geometrically increasing probability distribution (P) for picking a transmission slot in the contention window. Nodes which are executing IBA scheme pick a slot in the range of $(1, \mathrm{CW})$ with the probability distribution P. Here, CW is contention window and its value is fixed. More information on CW we will present in the later section of this paper. Figure 2 shows the probability distribution P. The higher slot numbers have higher probability to get selected by nodes compared to lower slot numbers. In physical meaning we can explain this as: at the start node select a higher slot number for its

\footnotetext{
${ }^{2}$ In rest of the paper we keep using terms 'DEV' and 'node' interchangeably.
} 
CW by estimating large population of active nodes $(\mathrm{N})$ and keep sensing the channel status. If no nodes transmits in the first or starting slots then each node adjust its estimation of competing nodes by multiplicatively increasing its transmission probability for the next slot selection cycle. Every node keeps repeating the process of estimation of active nodes in every slot selection cycle and allows the competition to happen at geometrically-decreasing values of $\mathrm{N}$ all within the fixed contention window (CW).

In contrast to the probability distribution $\mathrm{P}$, in uniform distribution, as shown in fig. 2 , all the contending nodes have the same probability of transmitting in a randomly chosen time slot. From fig. 2 we can depict that when the population of competing nodes $(\mathrm{N})$ is large, most of the nodes will choose medium to high slot numbers as their CW and a very few nodes will choose low slot numbers, hence, a collision-free transmission will take place in a low slot number. When $\mathrm{N}$ is medium, most nodes will choose higher slot numbers and a collision- free transmission will take place in a medium slot number. Similarly, when $\mathrm{N}$ is small, a collision free transmission will take place in a high slot number. Thus for any value of $\mathrm{N}$, and for any fast change in $\mathrm{N}$, a collision free transmission can take place. If only one node gets the chance to select the contention slot within the fixed CW, it will transmit in that slot. While other nodes will select new random contention slots for next competition to win channel medium, regardless of success or failure of transmission of winner node. Here it is worth to note that IBA scheme doesn't use timer suspension like in IEEE 802.11 to save energy and reduce latency in case of a collision. The only problem with the IBA is fairness, however, for WPAN MAC, especially, during CAP fairness is not an issue as every node don't have request packet for PNC for every time. For the general wireless communication scenario we need fairness mechanism, which we left to our future research investigation.

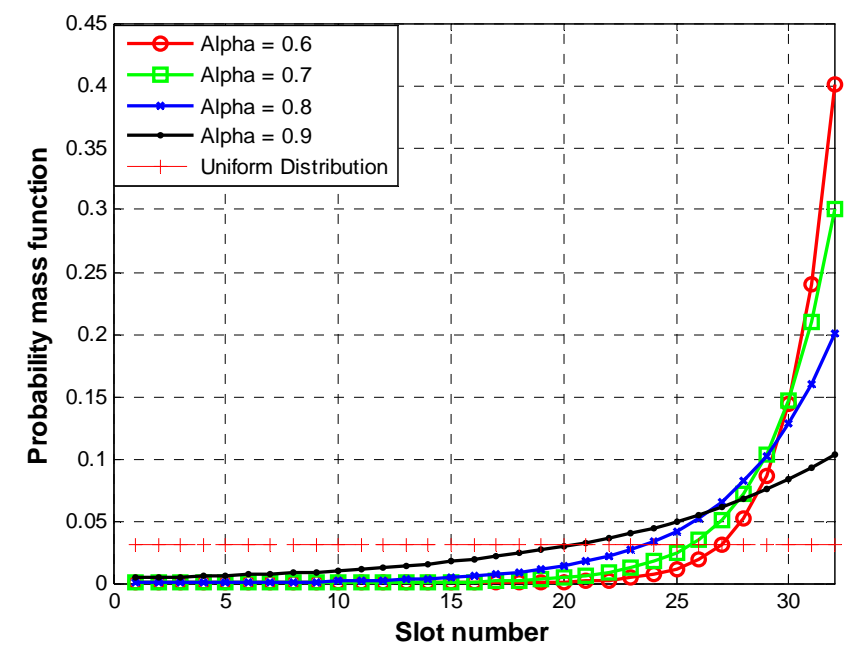

Figure 2. Difference between uniform and truncated geometric distributions

Lets say each node picks a slot $t_{r} \in[1, C W]$ using the probability distribution $P$. Then slot $t_{r}$ defines as an idle slot if no nodes choose it, and also as a collided slot if more than one node chooses it. A node can get the chance to transmit if it is the only node to choose slot $t_{r}$. 


\section{Macrothink}

As we mentioned earlier IBA uses a truncated, increasing geometric distribution, as presented in [3], and is given by

$$
p=\frac{(1-\alpha) \alpha^{C W}}{1-\alpha^{C W}} \alpha^{-t_{r}} \text { for } t_{r}=1, \ldots, C W,
$$

where $0<\alpha<1$ is a distribution parameter $^{3}$. In this range of $\alpha, p$ increases exponentially with $t_{r}$, so the later slots have higher probability. Using these probability distributions we calculated the probability of success and plotted as shown in figure 3. For our calculation we assume the values of different parameters as shown in table 1.

Table 1. Parameter's Value

\begin{tabular}{|c|c|}
\hline Parameters & Values \\
\hline$N$ & $1 \sim 256$ \\
\hline$\alpha$ & {$[0.7 \sim 0.9]$} \\
\hline$C W$ & {$[16,32,64]$} \\
\hline
\end{tabular}

As shown in fig. 3 the probability of a successful transmission is constantly high for a large number of contenders in case of $\mathrm{P}$ distribution. Also it outperformed the uniform distribution as shown in fig. 3. Figure 4 shows the degrade performance (below $70 \%$ of the value of $\mathrm{P}$ ) of $\mathrm{P}$ distribution when the true number of contenders $(\mathrm{N})^{4}$ exceeds 100 , and CW set to 16 slots. This degradation happens because the initial slots start to get picked by more than one station, hence, increase in probability of collisions. Figure 3 shows the results with different values of CW and its effect on the probability of success, and superiority of $\mathrm{P}$ distribution over uniform distribution as well.

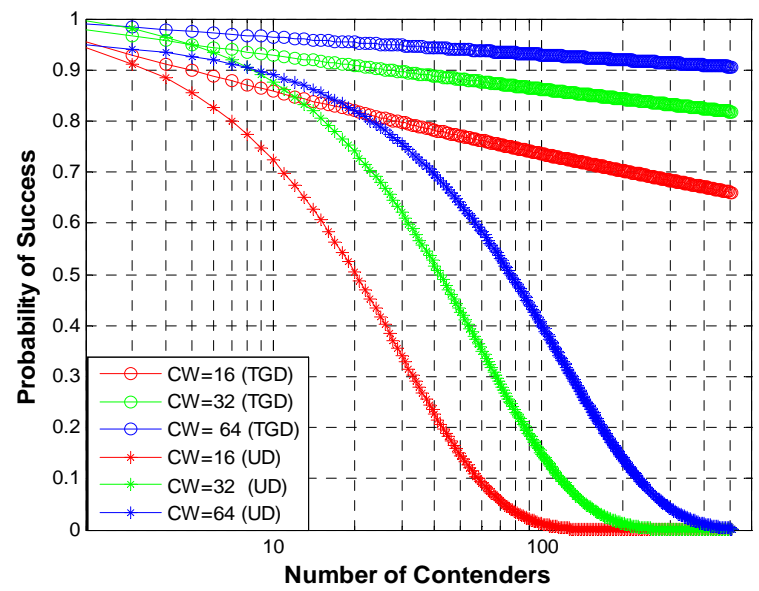

Figure 3. Number of contenders vs. Probability of success

\footnotetext{
${ }^{3}$ We can choose any number of nodes according to the given data to get an estimated value of $\alpha$.

${ }^{4}$ Here, we choose around 100 nodes according to [8], as the maximum number of contending nodes during a CAP frame.
} 


\section{Performance Evaluation}

In this subsection we present the performance analysis of BEB and IBA in terms of channel efficiency, medium access delay and energy-efficiency. For the performance analysis we carried out simulation in Matlab [9]. During a CAP nodes will send only request packets to PNC and PNC will assign them available slots in following CTA cycle. This assumption is reasonable as most of the data packet transmissions carried out in CTA slots. As we mentioned earlier here our main aim to evaluate the performance of both backoff methods, we limit our research only for CAP duration. The channel is an ideal and introducing no errors to the reception of a packet other than collision. Also, capture effect is not considered.

The BA performed in a time-slotted fashion. A node attempts to attain the access the channel only at the beginning of a slot. Furthermore, all nodes are well synchronized in time slots and propagation delay is negligible compared to the length of an idle slot.

Here, we define network load in terms of the number of nodes that are contending for the access medium. Another approach is to consider total arrival packet rate to the network as an offered load. The main parameters for our simulation are based on [10] and listed in table 2. For calculating the energy consumption in nodes we choose ratio of idle: listen: transmit as $1: 1: 1.5$, as measured in [11]. For the simulation results we doesn't consider the technology adopted at the Physical layer, however the physical layer determines some network parameter values like inter-frame spaces. Whenever necessary we choose the values of the physical layer dependent parameters by referring to [10]. For the "traditional” MAC protocol maximum retry limit is set to $3(\mathrm{~m}=3)$ according to IEEE 802.15.3 standard. The results obtained here are the average values of our collected data.

Figure 4 shows the normalized channel efficiency of BEB and IBA schemes. Here, we define the channel efficiency as ratio of useful channel time to send one request packet to total time required by a node to transmit a request packet, and is given by

$$
\eta=\frac{\text { Request Packet Time }}{\text { Request Packet Trasmission Time }}
$$

Table 2. Simulation Parameters

\begin{tabular}{|c|c|}
\hline Parameters & Values \\
\hline SIFS & 2.5 usec \\
\hline MIFS & 1 usec \\
\hline Preamble and PLCP Header & 9 usec \\
\hline CW $_{\min }$ & 8 \\
\hline Request Packet size & 24 Bytes \\
\hline Nodes & $5 \sim 100$ \\
\hline Data Rate & $1 \mathrm{Gbps}$ \\
\hline Control Signal Rate & $48 \mathrm{Mbps}$ \\
\hline
\end{tabular}




\begin{tabular}{|c|c|}
\hline Transmitting Energy & $50 \times 10^{-6} \mathrm{~J} / \mathrm{Bit}$ \\
\hline Idle/listening Energy & $75 \times 10^{-6} \mathrm{~J} / \mathrm{Bit}$ \\
\hline
\end{tabular}

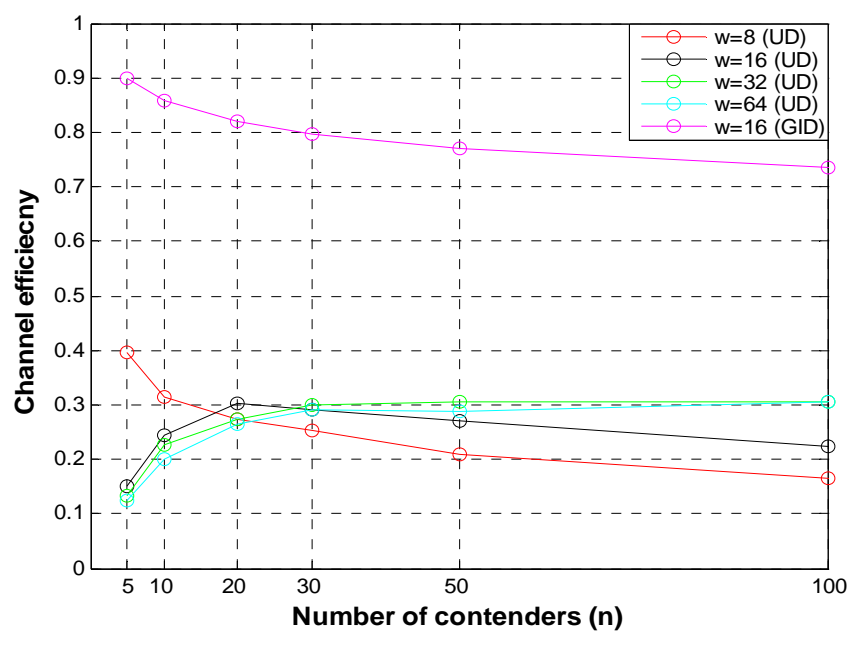

Figure 4. Number of contenders vs. Channel efficiency

As we have described in previous section channel efficiency is mostly depends on number of active nodes and contention window size. As shown in figure 4, BEB scheme is plotted for different value of $\mathrm{CW}$ and IBA is plotted for fixed value of $\mathrm{CW}=16^{5}$. For $\mathrm{BEB}$ scheme, results with $\mathrm{CW}=8$ and 16 gives high channel efficiency values at lower number of nodes. The reason is very obvious, less collision and low waiting time in backoff procedure, and as number of contenders increases channel efficiency start decreasing. For CW=32 and 64, at first graphs shows lower channel efficiency, and then increases to higher values, and later maintains nearly constant values. This is because at first $\mathrm{CW}$ is too big for given number of nodes and later it adjusted with the number of nodes, hence nearly constant collision and waiting time in backoff procedure. In contrast to BEB, IBA maintains high channel efficiency due to its unique quality of collision avoidance among the competing nodes. In IBA most of the nodes choose higher contention slots while very few nodes selects lower contention slots, hence less or no collision and low waiting time in backoff procedure.

Figure 5 shows the average medium access delay performances of BEB and IBA. Here, medium access delay is defined as the time elapsed between the generation of a request packet and its successful reception. In BEB scheme, as a large number of stations attempt to access the medium, more collision occur, the number of retransmissions increases and the nodes suffer longer delays. The BEB scheme is plotted for different values of CW.

\footnotetext{
5 This value is selected from our previous work [12] and [2]. Furthermore, it is the best value in trade off between channel efficiency and energy efficiency in WPAN MAC protocol.
} 


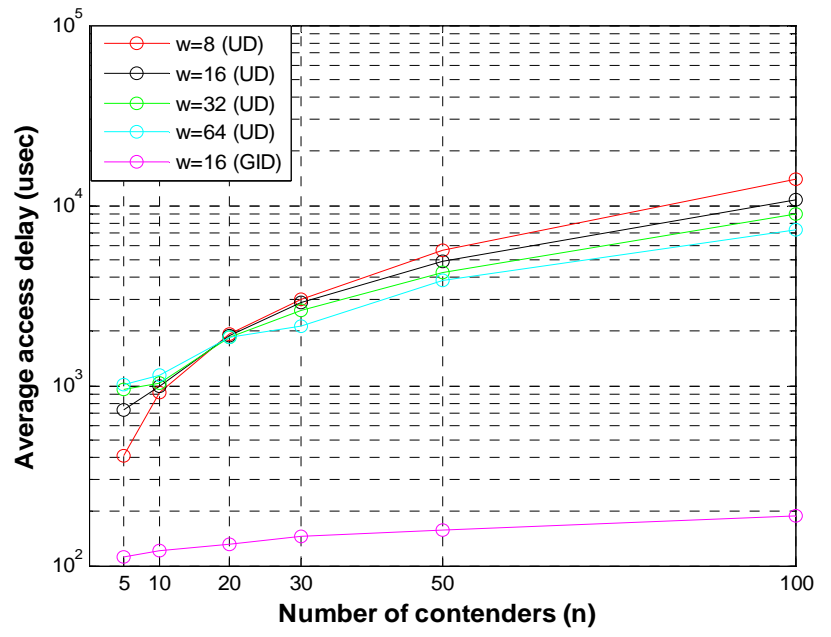

Figure 5. Number of contenders vs. Average access delay

In IBA, as we expected access delay is very low compared to BEB. This is because of low or no collision and less idle waiting time in backoff procedure. Figure 6 illustrates the impact of CW on average energy efficiency of BEB and IBA schemes.

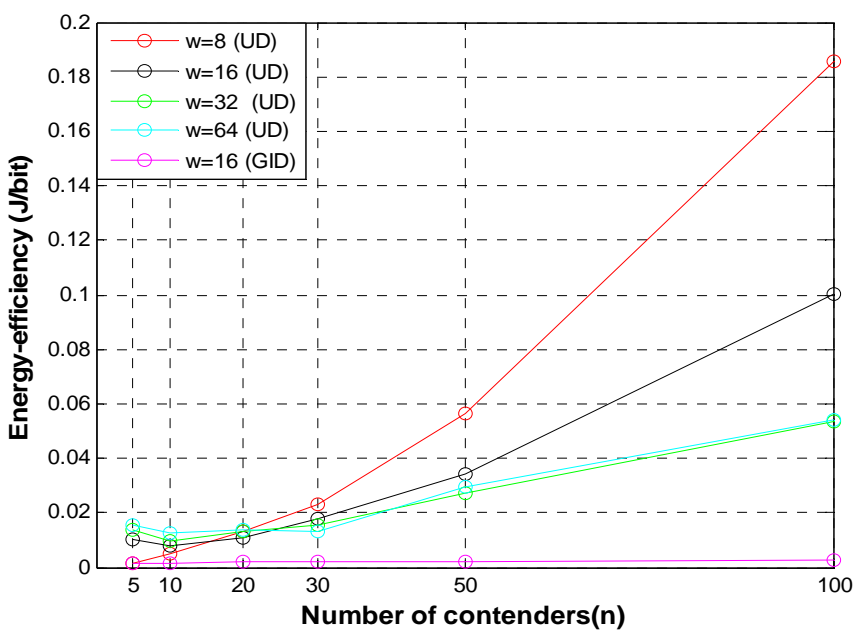

Figure 6. Number of contenders vs. Energy efficiency

We define the energy efficiency as the energy required to successfully transmit one bit of request data. From figure 6 we can see that for small value of CW in BEB scheme waste more energy as collision and number of retransmission increases. As CW value increases the collision probability and the number of retransmissions reduces, hence less energy waste. In contrast IBA wastes very less energy due to its unique characteristics of collision avoidance. From all aforementioned results we can see the superiority of IBA over BEB. Accepting IBA as backoff scheme can increase the overall performance of WPAN MAC protocol to a large extends. 


\section{Conclusions}

In this paper we applied an IBA scheme to WPAN MAC to enhance its performance. Our results show the performance analysis of BEB and IBA in terms of channel efficiency, medium access delay and energy-efficiency. Furthermore, we show the logical argument and superiority of IBA against the BEB based traditional algorithm. In near future, we would like to extend our work to more general wireless networks scenarios with fairness mechanism.

\section{Acknowledgement}

We would like to thanks anonymous reviewers for their valuable comments, and helping us to further improve the quality of this paper.

\section{References}

[1] P802.15.3/D17, “(C/LM) Standard for Telecommunications and Information Exchange Between Systems - LAN/MAN Specific Requirements - Part 15.3: Wireless Medium Access Control (MAC) and Physical Layer (PHY) Specifications for High Rate Wireless Personal Area Networks”, Feb. 2003

[2] S.Mehta and K.S.Kwak, “ Capacity Analysis of IEEE 802.15.3 MAC Protocol ,” Technical Report-4, UWB Research Center, Korea, May/June 2008.

[3] Y. C. Tay, K. Jamieson, and H. Balakrishnan, “ Collision-Minimizing CSMA and Its Applications to Wireless Sensor Networks," IEEE Journal on Selected Areas of Communications (JSAC), Vol.22, No..6, August 2004. pp.1048-1057.

[4] B. J. Kwak, N.O.Song, and L. E. Miller,” Performance Analysis of Exponential Backoff,” IEEE/ACM Transactions on Networking, VOL. 13, NO. 2, April 2005.

[5] C. Hu, H. Kim, and J. C. Hou, "An Analysis of the Binary Exponential Backoff Algorithm in Distributed MAC Protocols," Report No. UIUCDCS-R-2005-2599 UILU-ENG-2005-1794. July 2005.

[6] Y. Ling and D.Meng, “ Study on Improved Truncated Binary Exponential Back-off Collision Resolution Algorithm," in International Journal of Computer Science and Network Security (IJCSNS), VOL. 6 No.11, November 2006.

[7] J. Li, X. Zeng, and Q. Su, “ Performance Investigation of Backoff Algorithms in Multihop Wireless Networks," in the proceeding of 9th International Conference for Young Computer Scientists, November 2008.

[8] Y-H Tseng, H-K Wu, and G-H Chen, “ Maximum Traffic Scheduling and Capacity Analysis for IEEE 802.15.3 High Data rate MAC Protocol”, in proc. of IEEE Vehicular Technology Conference’03, vol.3 pp. 1678-1682,Oct. 2003

[9] The Mathsworks-Matlab, http://www.mathworks.com/products/matlab/

[10]H. Harada et. al., “ IEEE P802.15 Working Group for Wireless Personal Area Networks (WPANs)”, IEEE P802.15 Working Group for Wireless Personal Area Networks (WPANs) 
DOC: IEEE 802.15-07-0761-05-003c, July 2007.

[11]M. Stemm, R.H. Katz, “ Measuring and reducing energy consumption of network interfaces in hand-held devices,” IEICE Transactions on Communications E80-B (8) (1997) pp.1125-1131.

[12]S.Mehta and K.S.Kwak, “A GCSMA Based Contention Scheme for IEEE 802.15.3 Networks,” Technical Report-5, UWB Research Center, Korea, March/April 2009. 